\title{
REVIEW
}

\section{Clinical and histopathological risk factors for distant metastasis in head and neck cancer patients}

\author{
Fattori di rischio clinici e istopatologici per metastasi a distanza in pazienti \\ con carcinoma della testa e del collo
}

\begin{abstract}
Carlos Miguel Chiesa-Estomba1,2, Jerome R. Lechien²,3, Tareck Ayad²,4, Christian Calvo-Henriquez²,5, José Ángel González-García ${ }^{1}$, Jon Alexander Sistiaga-Suarez ${ }^{1}$, Didier Dequanter ${ }^{6}$, Nicolas Fakhry²,7, Gebeyehu Melesse ${ }^{8}$, Cesare Piazza ${ }^{9}$

${ }^{1}$ Department of Otorhinolaryngology - Head and Neck Surgery, Hospital Universitario Donostia, San Sebastian, Spain; ${ }^{2}$ Head \& Neck Study Group of Young-Otolaryngologists of the International Federations of Oto-rhino-laryngological Societies (YO-IFOS); ${ }^{3}$ Department of Human Anatomy and Experimental Oncology, University of Mons, Mons, Belgium; ${ }^{4}$ Division of Otolaryngology Head and Neck Surgery, Centre Hospitalier de l'Université de Montréal, Montreal, Canada; ${ }^{5}$ Department of Otolaryngology, Hospital Complex of Santiago de Compostela, Santiago de Compostela, Spain; ${ }^{6}$ Department of Otolaryngology - Head and Neck Surgery, Centre Hospitalier Universitaire Saint-Pierre, Brussels, Belgium; ${ }^{7}$ Department of Otolaryngology - Head and Neck Surgery, Universitary Hospital of la Conception, Marseille, France; ${ }^{8}$ Department of Otolaryngology - Head and Neck Surgery, Bahir Dar University, Tibebe Ghion Specialised Hospital, Bahir Dar, Ethiopia; ${ }^{9}$ Department of Otorhinolaryngology, Maxillofacial and Thyroid Surgery, Fondazione IRCCS, National Cancer Institute of Milan, University of Milan, Milan, Italy
\end{abstract}

\section{SUMMARY}

The incidence of distant metastasis (DM) in head and neck squamous cell cancer (HNSCC) is relatively low. Multiple risk factors have been described for development of DM at baseline and after treatment. However, to date, there is no meta-analysis or systematic review investigating the relationships between clinical and histopathological factors and the appearance of DM in HNSCC patients. Among 1,272 eligible articles, 23 met inclusion criteria for qualitative analysis, and 6 for quantitative analysis. The meta-analysis on 5,353 patients showed that hypopharyngeal site, T3-T4 categories, extranodal extension, positive lymph node size $>6 \mathrm{~cm}$, locoregional failure after previous treatment(s) and poor differentiation all significantly increase the risk of DM. According to our results, patients with the above-mentioned clinical and histopathological risk factors should be considered at high risk for DM and therefore submitted to strict pre-treatment assessment and undergo careful post-therapeutic follow-up.

KEY WORDS: distant metastasis, head and neck, cancer, risk factors

\section{RIASSUNTO}

L'incidenza di metastasi a distanza nel carcinoma squamocellulare della testa e del collo è relativamente bassa. Sono stati descritti molteplici fattori di rischio per lo sviluppo di metastasi sistemiche, sia al momento della diagnosi che dopo il trattamento. In ogni caso, ad oggi, non esiste una meta-analisi o una revisione sistematica che indaghi i rapporti tra fattori clinico-istopatologici e la comparsa di metastasi a distanza nei pazienti con carcinoma squamocellulare della testa e del collo. Tra i 1,272 articoli utili, 23 presentavano i criteri di inclusione per un'analisi qualitativa e 6 erano adatti ad un'analisi di tipo quantitativo. La meta-analisi, condotta su un totale di 5,353 pazienti, ha mostrato come la sede ipofaringea, le categorie T3-T4, l'estensione extra-nodale, linfonodi metastatici di diametro $>6 \mathrm{~cm}$, il fallimento loco-regionale dopo pregressi trattamenti e la scarsa differenziazione aumentino tutti in modo significativo il rischio di metastasi a distanza. In base ai nostri risultati, quindi, i pazienti con i fattori di rischio clinici e istopatologici sopra citati dovrebbero essere considerati ad alta probabilità di sviluppare metastasi a distanza e, pertanto, sottoposti ad una rigorosa valutazione pre-trattamento, così come ad un attento follow-up.

PAROLE CHIAVE: metastasi a distanza, testa e collo, cancro, fattori di rischio
Received: May 23, 2020

Accepted: June 6, 2020

Correspondence

Carlos Miguel Chiesa-Estomba

Department of Otorhinolaryngology - Head and

Neck Surgery, Hospital Universitario Donostia, Calle Doctor Begiristain \#1. CP. 20014, San Sebastian Donosti, Spain

Tel. +34 635793435

E-mail: chiesaestomba86@gmail.com

Funding

None.

Conflict of interest

The Authors declare no conflict of interest.

How to cite this article: Chiesa-Estomba CM, Lechien JR, Ayad T, et al. Clinical and histopathological risk factors for distant metastasis in head and neck cancer patients. Acta Otorhinolaryngol Ital 2021;41:6-17. https://doi. org/10.14639/0392-100X-N0879

(c) Società Italiana di Otorinolaringoiatria e Chirurgia Cervico-Facciale

\section{cc) (1) $(9)$}

This is an open access article distributed in accordance with the CC-BY-NC-ND (Creative Commons Attribution-NonCommercial-NoDerivatives 4.0 International) license. The article can be used by giving appropriate credit and mentioning the license, but only for non-commercial purposes and only in the original version. For further information: https:// creativecommons.org/licenses/by-nc-nd/4.0/deed.en 


\section{Introduction}

In head and neck squamous cell carcinoma (HNSCC), the main sites of distant metastases (DM) are the lung, bone and liver, accounting for approximately $70-85 \%, 15-39 \%$ and $10-30 \%$ of events, respectively ${ }^{1}$. The incidence of DM in HNSCC is, however, relatively low, with a reported prevalence of clinically identified distant localisations at diagnosis ranging from $3 \%$ to $50 \%{ }^{2-18}$.

HNSCC patients with DM are generally candidates for palliative treatment since no systemic therapy has curative potential in such a clinical scenario ${ }^{18}$. As a consequence, the reported median overall survival (OS) in the literature is around 10 months ${ }^{19}$, and extensive locoregional treatments are universally believed to be futile for their modest (if any) improvement in OS.

Multiple studies have evaluated the main risk factors for development of DM at baseline and/or after treatment of $\mathrm{HN}$ SCC 14,17,21-24. These generally include the presence of $\geq 3$ neck lymph nodes metastases ${ }^{7,25}$, radiological or histological extranodal extension (ENE) ${ }^{22,25,27-29}$, low jugular positive lymph nodes ${ }^{25,30}$, nodal metastases $\geq 6 \mathrm{~cm}$ in size ${ }^{7,25,31}$, bilateral lymph nodes metastases ${ }^{7,25}$, presence of a second primary $\mathrm{HNSCC}^{5}$, regional recurrence ${ }^{25}$, primary tumour of the pharynx ${ }^{4,7,22,30,31}$ and advanced $\mathrm{T}$ categories ${ }^{4,31}$.

Using the Surveillance, Epidemiology, and End Results (SEER) database, Kuperman et al. ${ }^{11}$ conducted a cohort study on 27,877 patients aimed at identification of risk factors for DM at the time of HNSCC diagnosis. The authors identified hypopharyngeal cancer, $\mathrm{N} 3$ category and size of the primary tumour $>4 \mathrm{~cm}$ as the most important risk factors for DM. In the same way, Liu et al. ${ }^{18}$ used the National Cancer Database (NCDB) data on 151,730 patients to identify patterns of DM in HNSCC at the time of diagnosis. In that study, the authors identified age at diagnosis, ethnicity, HPV status, tumour grade, T4 and N3 categories as the most predictive variables for DM. Interestingly, high-risk HPV status was associated with a lower proportion of $\mathrm{DM}^{18}$. The design in these two studies was population-based and the findings help in partially understanding the behaviour of DM in HNSCC. However, to date, no systematic review or meta-analysis has been carried out to investigate the relationships between clinical-histopathological risk factors and DM in HNSCC patients. The objective of this work is, therefore, to better understand the available evidence in the contemporary literature about the clinical and histopathological risk factors for DM in HNSCC patients after treatment with curative intention.

\section{Materials and methods}

This systematic review used Population Intervention Comparison and Outcome (PICO) modeling and followed the guidelines proposed by the Preferred Reporting Items for Systematic reviews and Meta-Analyses (PRISMA) statement.

\section{Population and inclusion/exclusion criteria}

Inclusion criteria consisted of clinical series comprehensively reporting clinical (age, pharyngeal site, $\mathrm{T}$ and $\mathrm{N}$ categories, neck lymph nodes status, local and regional control or failure) and histopathological data (ENE, p16 status, lymph node size $>6 \mathrm{~cm}$, and number of lymph nodes) of HNSCC patients without DM at presentation to evaluate the appearance of DM during follow-up after treatment. Studies that evaluated the association between relevant clinical or histological factors with the appearance of DM through univariate and multivariate analysis were considered. Case reports, case series and expert opinion papers were excluded, as well as articles focusing on non-HNSCC patients, with different or multiple histology, and absence of detailed clinical and/or histopathological information.

\section{Intervention and comparison}

Intervention and comparison groups were defined according to risk factors: T1-T2 versus T3-T4, tobacco use, p16 status, ENE, positive versus negative lymph nodes, $\mathrm{N}$ category, age $<60$ versus $>60$ years, lymph node size $<6 \mathrm{~cm}$ versus $>6 \mathrm{~cm}$, presence of $<3$ versus $\geq 3$ positive lymph nodes, and locoregional control versus locoregional failure. The degrees of histological differentiation (well, moderate, and poor) were also compared.

\section{Outcomes}

The primary outcome evaluated in this study was risk of developing DM in HNSCC patients according to the abovementioned clinical and histopathological factors.

\section{Search strategy}

A systematic search of electronic databases was conducted on MEDLINE/PUBMED, Google Scholar, Ovid Medline, Embase, Scopus, Cochrane Database of Systematic Reviews, Cochrane Central Register of Controlled Trials, and the Database of Abstracts of Reviews of Effects. Papers in English language from January 1960 to July 2019 were included. The following keywords were used: (["distant metastasis" OR "head and neck cancer" OR "distant metastasis workup" OR "follow-up"]). Titles and abstracts were screened by two investigators (CMCE and JASS) to discard irrelevant publications. For each study, the following information were extracted: author, year of publication, number of patients evaluated, and clinical and histopathological characteristics. 


\section{Assessment of quality}

Methodological quality of identified studies was appraised using the Oxford Centre for Evidence-Based Medicine (OCEBM) Levels of Evidence. According to this, prospective or retrospective studies (Grading A-B) were included. Concerning assessment of risk of bias in individual cohort studies, the risk of bias in non-randomised studies of interventions tool (ROBIN-I) was used.

\section{Statistical analysis}

An inverse variance meta-analysis of selected studies with an odds ratio (OR) comparing information about tumour location, $\mathrm{T}$ and $\mathrm{N}$ categories, number of positive lymph nodes, age, histological differentiation, site of DM, ENE, locoregional control and recurrence rate was performed. The comparison was made using Cochrane Review Manager 5.3 (Nordic Cochrane Centre, Cochrane Collaboration, 2014, Copenhagen, Denmark). Heterogeneity was checked using the Q-test and I2 test. The I2 value was $>50 \%$, and the random-effects model was more appropriate, in which both random variation within studies and variations among the different studies were incorporated.

Cochrane Review Manager uses the Mantel-Haenszel method for calculating the heterogeneity and statistic is incorporated to calculate the summary of adjusted OR under the random-effects model. The pooled OR with $95 \%$ confidence interval $\left(\mathrm{CI}_{95 \%}\right)$ is given for the random-effects model.

In addition, a chi-square test with Yates correction for continuity was applied with a 2-tailed $p$ value for comparison of proportions according to gender and histological differentiation from independent samples. A p $<0.05$ was considered statistically significant.

\section{Overview of clinical and epidemiological characteristics}

In addition to the meta-analysis, we extended our research to all trials carried out during the past six decades with the aim to assess the clinical significance of HPV, impact of radiological development and type of organs affected by DM in HNSCC patients. Thus, in a second step, the two main investigators (CMCE and JASS) performed the same extraction and analysis of data for all controlled or uncontrolled, prospective, or retrospective studies conducted. The intent of this analysis was to allow robust overview of the characteristics of DM in HNSCC patients, which may allow elaboration of recommendations and perspectives.

\section{Results}

The literature search retrieved a total of 1,272 manuscripts, 72 of which met eligibility for full text review. Only 23 papers were included in the systematic review and com- mented in the Discussion section, while 6 (accounting for 5,353 patients) were included in the meta-analysis (Fig. 1). According to the OCEBM grading system, all the studies were rated as Level B (2c) and the overall bias according to ROBIN-I was considered to be at low to moderate risk in all studies. Demographic data of the studies included are summarised in Table I. A total of 4,814 (89.9\%) patients were males and $535(10.1 \%)$ females. DM were present in $516(9.6 \%)$ patients and were more common in men (Tab. I). Variables between each group were only partially comparable, which made challenging every comparison between cohorts due to the heterogeneity of data reported in each study (Tab. II). Concerning the primary site, the hypopharynx was related to a higher risk of DM development (18.7\%) (Tab. II). DM were more common in T4 tumors $(17.1 \%)$, N3 category $(17.1 \%)$, and in tumours with poorly differentiated histology $(29.3 \%)$. The lung was the most commonly affected organ $(61.8 \%)$. Additional data on $\mathrm{T}$ and $\mathrm{N}$ categories, histological differentiation and the organs affected by DM are reported in Tables III-V. Forest plots related to $\mathrm{T}$ and $\mathrm{N}$ categories, age, ENE, size and number of positive lymph node(s) and locoregional control (LRC) versus recurrence or treatment failure (R-F) are reported in Figures 2 and 3.

\section{Subgroups analysis}

\section{a) $T$ category}

When stratifying patients according to T category (T1$\mathrm{T} 2$ versus $\mathrm{T} 3-\mathrm{T} 4)$, the incidence of $\mathrm{DM}$ was $4.85 \%$ $\left(\mathrm{CI}_{95 \%} 4.05-5.65 \%\right)$ versus $14 \% \quad\left(\mathrm{CI}_{95 \%} 12.6-15.3 \%\right)$ ( $\mathrm{p}=0.0001)$. These differences were significantly in favour of T3-T4 categories as a risk factor for DM (OR 0.39; $\mathrm{CI}_{95 \%} 0.32-0.47 ; \mathrm{p}=0.001$ ) (Tab. III and Fig. 2A).

b) Age

When stratifying patients according to age $(<60$ versus $>60$-year-old), the incidence of DM was $9.4 \%$ $\left(\mathrm{CI}_{95 \%} 8.06-10.7 \%\right)$ versus $8.2 \% \quad\left(\mathrm{CI}_{95 \%} 7.05-9.35 \%\right)$ $(\mathrm{p}=0.173)$. These differences were not significant (OR $1.14 ; \mathrm{CI}_{95 \%} 0.92-1.43 \% ; \mathrm{p}=0.213$ ) (Tab. I and Fig. 2B).

c) Locoregional control

When stratifying patients according to LRC versus $\mathrm{R}-\mathrm{F}$, the incidence of DM was 5.9\% $\left(\mathrm{CI}_{95 \%} 4.7 \%-7.07 \%\right)$ in patients with favorable disease control versus $16.5 \%\left(\mathrm{CI}_{95 \%}\right.$ $13.9 \%-19.07 \%)$ in those with recurrence or treatment failure $(p=0.0001)$. These differences were significant with the absence of locoregional control as a risk factor for DM (OR 0.35; $\mathrm{CI}_{95 \%} 0.26-0.47 ; \mathrm{p}=0.0001$ ) (Fig. 2C).

d) $N$ category

When stratifying patients according to $\mathrm{N}$ category (N0 versus $\mathrm{N}+)$, the incidence of $\mathrm{DM}$ was $3.27 \%\left(\mathrm{CI}_{95 \%} 2.62\right.$ $3.92 \%)$ versus $19.4 \%\left(\mathrm{CI}_{95 \%} 17.7-21.05 \%\right)(\mathrm{p}=0.0001)$. 


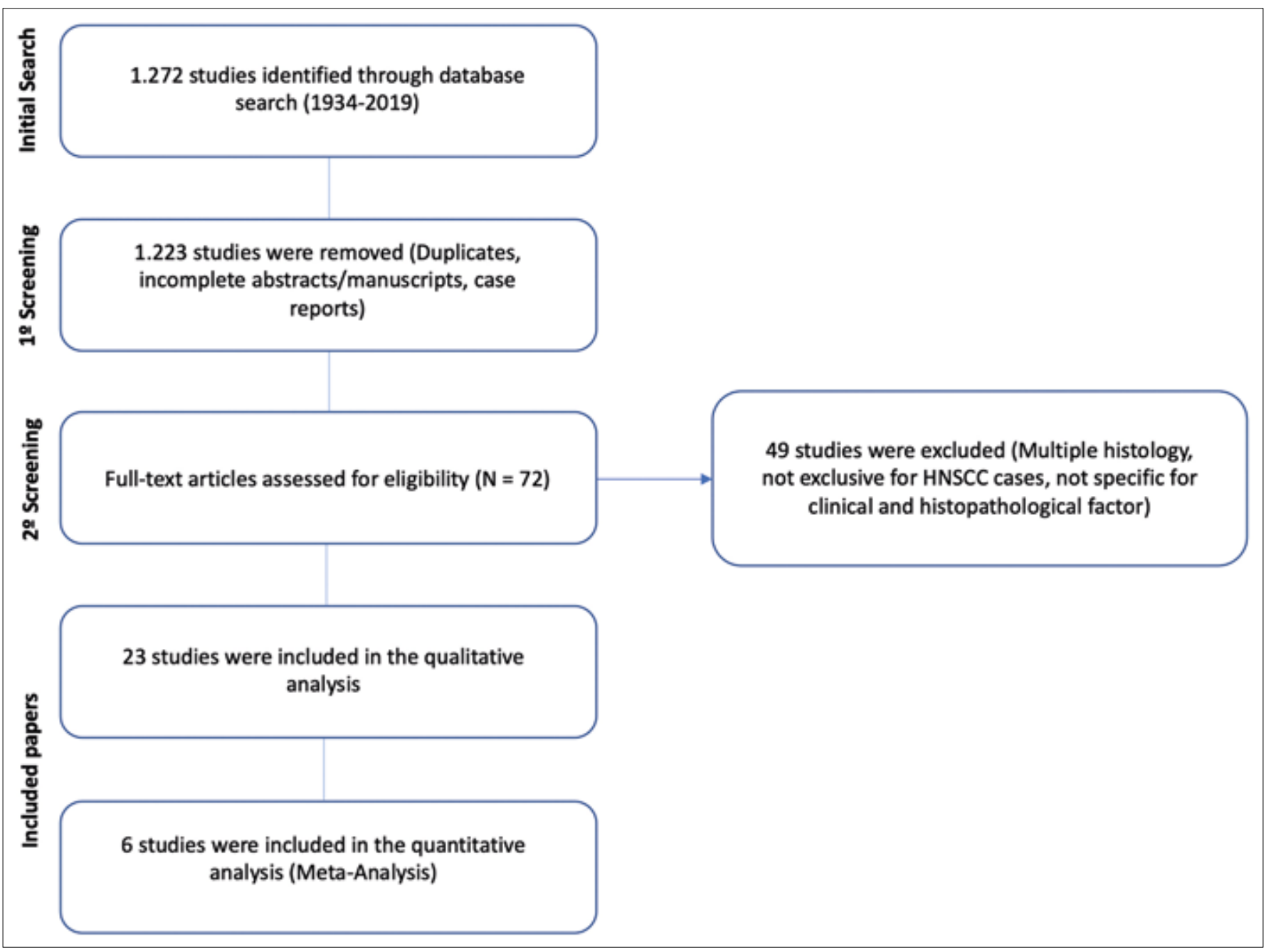

Figure 1. Algorithm for selection of studies.

Table I. Demographics, overall, and gender-related distant metastasis rates in the 6 studies included in the meta-analysis.

\begin{tabular}{|c|c|c|c|c|c|c|c|c|c|}
\hline Authors & $\begin{array}{l}\text { Type of } \\
\text { study }\end{array}$ & Period & $\begin{array}{l}\text { Mean } \\
\text { age }\end{array}$ & $\begin{array}{l}\text { Patients } \\
\text { included }\end{array}$ & Male & Females & $\begin{array}{l}\text { Overall DM } \\
\text { rate }\end{array}$ & $\begin{array}{l}\text { DM rate } \\
\text { in men }\end{array}$ & $\begin{array}{l}\text { DM rate } \\
\text { in women }\end{array}$ \\
\hline Leemans (1993) ${ }^{28}$ & $\mathrm{R}$ & 1973-1986 & 62 & 281 & $77.5 \%$ & $22.5 \%$ & $9.2 \%$ & NA & NA \\
\hline León $(2000)^{4}$ & $\mathrm{R}$ & 1984-1996 & NA & 1244 & $88.1 \%$ & $11.9 \%$ & $5.1 \%$ & $5.5 \%$ & $3.5 \%$ \\
\hline Garavello (2006) ${ }^{5}$ & $\mathrm{R}$ & 1981-1998 & 62 & 1972 & $94 \%$ & $6 \%$ & $9.1 \%$ & $9.1 \%$ & $10.2 \%$ \\
\hline Li (2009) 6 & $\mathrm{R}$ & $1990-2000$ & NA & 391 & $80 \%$ & $20 \%$ & $11.2 \%$ & $12.4 \%$ & $7.6 \%$ \\
\hline Coca-Pelaz (2011) ${ }^{7}$ & $\mathrm{R}$ & 1999-2006 & 60.2 & 443 & $97.2 \%$ & $2.8 \%$ & $13.9 \%$ & $13.2 \%$ & $25 \%$ \\
\hline Duprez (2017) ${ }^{22}$ & $\mathrm{R}$ & $1996-2015$ & 61 & 1022 & $89.9 \%$ & $10.1 \%$ & $13.7 \%$ & NA & NA \\
\hline Total & & & 62 & 5353 & $89.9 \%$ & $10.1 \%$ & $9.6 \%$ & $8.8 \%$ & $8.2 \%$ \\
\hline
\end{tabular}

R: retrospective; NA: not available; DM: distant metastasis.

These differences were significantly in favour of a $\mathrm{N}+$ neck as a risk factor for DM (OR 0.16; $\mathrm{CI}_{95 \%} 0.13-0.21$; $\mathrm{p}=0.0001$ ) (Tab. III and Fig. 3A).

e) Extranodal extension

When stratifying $\mathrm{N}+$ patients according to ENE (nega- tive versus positive), the incidence of DM was $13.6 \%$ $\left(\mathrm{CI}_{95 \%} 11.2 \%-15.9 \%\right)$ versus $26 \%\left(\mathrm{CI}_{95 \%} 22.9 \%-29.06 \%\right)$ $(\mathrm{p}=0.0001)$. These differences were significantly in favour of the presence of ENE as a risk factor for DM (OR $0.52 ; \mathrm{CI}_{95 \%}$ 0.40-0.67; $\mathrm{p}=0.0001$ ) (Fig. 3B). 
Table II. Distant metastasis rates according to primary sites.

\begin{tabular}{|c|c|c|c|c|c|c|c|c|c|}
\hline Authors & $\begin{array}{l}\text { Oral } \\
\text { cavity }\end{array}$ & Oropharynyx & Hypopharynx & Larynx & Glottis & Supraglottis & $\begin{array}{l}\text { Oropharynx } \\
\text { HPV-positive }\end{array}$ & CUP & Nasopharynx \\
\hline León (2000) ${ }^{4}$ & $0.8 \%$ & $7.3 \%$ & $18.7 \%$ & $4 \%$ & $1.2 \%$ & $8.4 \%$ & - & - & $11.9 \%$ \\
\hline Garavello (2006) ${ }^{5}$ & $2.9 \%$ & $10.4 \%$ & $16.7 \%$ & $9.2 \%$ & $8.7 \%$ & $10.1 \%$ & - & - & - \\
\hline Coca-Pelaz (2011) ${ }^{7}$ & $14.7 \%$ & $16.6 \%$ & $20.4 \%$ & $7.6 \%$ & NA & NA & - & - & - \\
\hline Duprez (2017) 22 & $15.3 \%$ & $12.6 \%^{a}$ & $20.5 \%$ & $9 \%$ & NA & NA & $22.2 \%^{\mathrm{b}}$ & $19.6 \%$ & - \\
\hline Total & $6.3 \%$ & $11.1 \%$ & $18.7 \%$ & $7.2 \%$ & NA & NA & - & - & - \\
\hline
\end{tabular}

CUP: carcinoma of unknow primary; NA, not available; ${ }^{a}$ These patients correspond to those without HPV testing; ${ }^{b}$ These patients correspond to those with known HPV-positive status.

Table III. Relationship between T, N categories and degree of histological differentiation with the rate of distant metastasis.

\begin{tabular}{|c|c|c|c|c|c|}
\hline Authors & TO & $\mathrm{T1}$ & T2 & T3 & $\mathrm{T} 4$ \\
\hline León (2000) ${ }^{4}$ & 0 & $0.2 \%$ & $4.9 \%$ & $7.8 \%$ & $13.7 \%$ \\
\hline Garavello (2006) ${ }^{5}$ & 0 & $0.3 \%$ & $7.8 \%$ & $12.6 \%$ & $20.9 \%$ \\
\hline LI (2009) 6 & 0 & $6 \%$ & $8 \%$ & $11.7 \%$ & $14.9 \%$ \\
\hline Coca-Pelaz (2011) ${ }^{7}$ & 0 & $5.7 \%$ & $11.6 \%$ & $14 \%$ & $17.6 \%$ \\
\hline Duprez (2017) 22 & $15.8 \%$ & $8 \%$ & $12.9 \%$ & $15.9 \%$ & $15.2 \%$ \\
\hline Total & $15.8 \%$ & $1.7 \%$ & $8.5 \%$ & $12 \%$ & $17.1 \%$ \\
\hline Authors & NO & N1 & N2 & N3 & \\
\hline León (2000) ${ }^{4}$ & $1.7 \%$ & $1.5 \%$ & $16.4 \%$ & $24.1 \%$ & \\
\hline Garavello (2006) & $2.5 \%$ & $21.9 \%$ & $23.7 \%$ & $29.4 \%$ & \\
\hline Coca-Pelaz (2011) ${ }^{7}$ & $4.7 \%$ & $9 \%$ & $23 \%$ & $33 \%$ & \\
\hline Duprez (2017) 22 & $5 \%$ & $11.2 \%$ & $8.5 \%$ & $28.8 \%$ & \\
\hline Total & $2.7 \%$ & $16.6 \%$ & $20.6 \%$ & $29.3 \%$ & \\
\hline Authors & Well (G1) & Moderately (G2) & $\begin{array}{c}\text { Poorly } \\
\text { (G3) }\end{array}$ & & \\
\hline León (2000) ${ }^{4}$ & $2.5 \%$ & $4.7 \%$ & $13.1 \%$ & & \\
\hline Garavello (2006) ${ }^{5}$ & $1 \%$ & $8.4 \%$ & $17.7 \%$ & & \\
\hline LI (2009) ${ }^{6}$ & $7.6 \%$ & $13.6 \%$ & $13.6 \%$ & & \\
\hline Coca-Pelaz $(2011)^{7}$ & $5 \%$ & $17 \%$ & $25 \%$ & & \\
\hline Duprez (2017) ${ }^{22}$ & $9 \%$ & $14 \%$ & $15.6 \%$ & & \\
\hline Total & $4.2 \%$ & $9.2 \%$ & $16.9 \%$ & & \\
\hline
\end{tabular}

Table IV. Organs affected by HNSCC distant metastases.

\begin{tabular}{|c|c|c|c|c|c|c|c|c|c|c|c|c|c|c|c|c|}
\hline Authors & Lung & Liver & Bone & Skin & $\begin{array}{l}\text { Multi- } \\
\text { ple DM }\end{array}$ & Parotid & $\begin{array}{c}\text { Exter- } \\
\text { nal au- } \\
\text { ditory } \\
\text { canal }\end{array}$ & $\begin{array}{c}\text { Soft } \\
\text { tissues }\end{array}$ & Brain & Pleura & $\begin{array}{c}\text { LN } \\
\text { outside } \\
\text { neck }\end{array}$ & $\begin{array}{c}\text { LN me- } \\
\text { diasti- } \\
\text { num }\end{array}$ & $\begin{array}{l}\text { LN ax- } \\
\quad \text { illa }\end{array}$ & $\begin{array}{c}\text { Omen- } \\
\text { tum }\end{array}$ & Spleen & $\begin{array}{c}\text { Adrenal } \\
\text { gland }\end{array}$ \\
\hline $\begin{array}{l}\text { León } \\
(2000)^{4}\end{array}$ & $51 \%$ & $4.6 \%$ & $12.5 \%$ & 0 & $31.2 \%$ & 0 & 0 & 0 & 0 & 0 & 0 & 0 & 0 & 0 & 0 & 0 \\
\hline Li (2009) ${ }^{6}$ & $48.7 \%$ & $7.3 \%$ & $2.4 \%$ & $2.4 \%$ & $26.8 \%$ & $2.4 \%$ & $2.4 \%$ & $2.4 \%$ & 0 & $2.4 \%$ & $2.4 \%$ & 0 & 0 & 0 & 0 & 0 \\
\hline $\begin{array}{l}\text { Coca-Pelaz } \\
(2011)^{7}\end{array}$ & $52.3 \%$ & $1.6 \%$ & $3.1 \%$ & $1.6 \%$ & $38 \%$ & 0 & 0 & $1.6 \%$ & $1.6 \%$ & 0 & 0 & 0 & 0 & 0 & 0 & 0 \\
\hline $\begin{array}{l}\text { Duprez } \\
(2017)^{22}\end{array}$ & $40 \%$ & $8.9 \%$ & $15.6 \%$ & $12.2 \%$ & 0 & 0 & 0 & $0.7 \%$ & $0.3 \%$ & $4.8 \%$ & $10.4 \%$ & $4 \%$ & $0.7 \%$ & $0.3 \%$ & $0.3 \%$ & $0.7 \%$ \\
\hline
\end{tabular}


Table V. Risk of bias in individual cohort studies ((ROBIN-I).

\begin{tabular}{|c|c|c|c|c|c|c|c|}
\hline Author & $\begin{array}{l}\text { Bias due to } \\
\text { confunding }\end{array}$ & $\begin{array}{l}\text { Bias in } \\
\text { selection of } \\
\text { participants } \\
\text { into the } \\
\text { study }\end{array}$ & $\begin{array}{c}\text { Bias in } \\
\text { classification } \\
\text { of } \\
\text { interventions }\end{array}$ & $\begin{array}{l}\text { Bias due to } \\
\text { deviations } \\
\text { from } \\
\text { intended } \\
\text { intervention }\end{array}$ & $\begin{array}{l}\text { Bias due to } \\
\text { missing data }\end{array}$ & $\begin{array}{c}\text { Bias in } \\
\text { measurement } \\
\text { of } \\
\text { outcomes }\end{array}$ & $\begin{array}{l}\text { Bias in } \\
\text { selection } \\
\text { of the reported } \\
\text { results }\end{array}$ \\
\hline Coca-Pelaz (2011) ${ }^{7}$ & No information & Low risk & Low risk & Low risk & Moderate risk & Moderate risk & Low risk \\
\hline Duprez (2017) 22 & No information & Low risk & Low risk & Low risk & Moderate risk & Moderate risk & Low risk \\
\hline León (2000) ${ }^{4}$ & No information & Low risk & Low risk & Low risk & Moderate risk & Moderate risk & Moderate risk \\
\hline Garavello (2006) ${ }^{5}$ & No information & Low risk & Low risk & Low risk & Moderate risk & Moderate risk & Low risk \\
\hline Li (2009) ${ }^{6}$ & No information & Low risk & Low risk & Low risk & Moderate risk & Moderate risk & Low risk \\
\hline Leemans (1993) 28 & No information & Low risk & Low risk & Low risk & Moderate risk & Moderate risk & Moderate risk \\
\hline
\end{tabular}

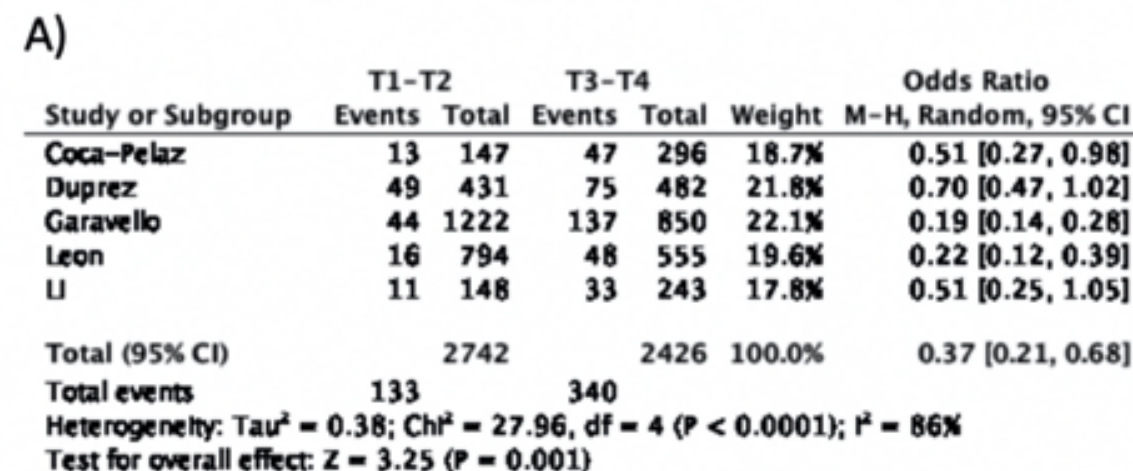

B)

\begin{tabular}{|c|c|c|c|c|c|c|}
\hline \multirow[b]{2}{*}{ Study or Subgroup } & \multicolumn{2}{|c|}{$<60$ years } & \multicolumn{2}{|c|}{$>60$ years } & \multirow[b]{2}{*}{ Weight } & \multirow{2}{*}{$\begin{array}{c}\text { Odds Ratio } \\
\text { M-H, Random, } 95 \% \mathrm{C}\end{array}$} \\
\hline & Events & Total & Events & Total & & \\
\hline $\begin{array}{l}\text { Coca-Pelaz } \\
\text { Garavello } \\
\text { Leon } \\
\text { U }\end{array}$ & $\begin{array}{l}27 \\
94 \\
30 \\
20\end{array}$ & $\begin{array}{l}229 \\
838 \\
561 \\
187\end{array}$ & $\begin{array}{l}33 \\
87 \\
34 \\
24\end{array}$ & $\begin{array}{r}214 \\
1134 \\
619 \\
204\end{array}$ & $\begin{array}{l}22.3 \% \\
34.4 \% \\
24.2 \% \\
19.1 \%\end{array}$ & $\begin{array}{l}0.73[0.42,1.27] \\
1.52[1.12,2.07] \\
0.97[0.59,1.61] \\
0.90[0.48,1.69]\end{array}$ \\
\hline Total $(95 \% \mathrm{Cl})$ & & 1815 & & 2171 & $100.0 \%$ & $1.05[0.73,1.51]$ \\
\hline $\begin{array}{l}\text { Total events } \\
\text { Heterogenelty: T }\end{array}$ & 171 & & $\begin{array}{r}178 \\
1, \text { df }=\end{array}$ & & & \\
\hline
\end{tabular}

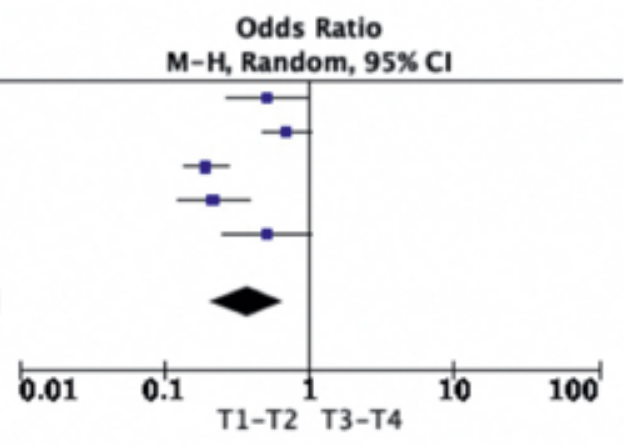

est for overall efiect: $z=3.25(P=0.001)$

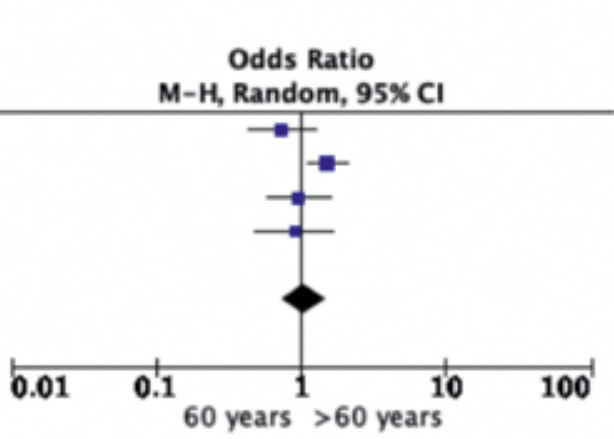

C)

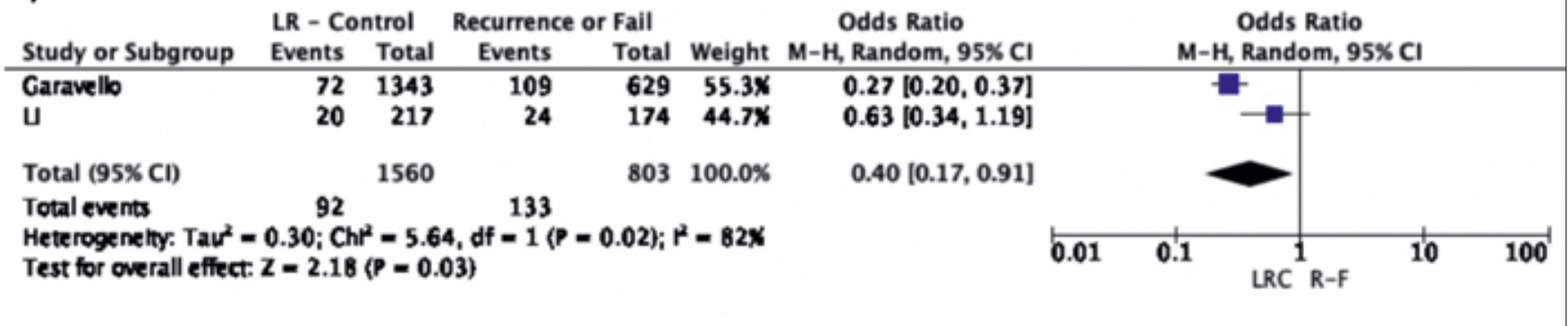

Figure 2. Forest plots showing the relationships between: (A) T-category; (B) Age; (C) Locoregional control and the appearance of DM. The experimental cohort was represented by patients with T1-T2 tumours and locoregional control, aiming to demonstrate the protective effect of these factors against the appearance of DM. 
A)

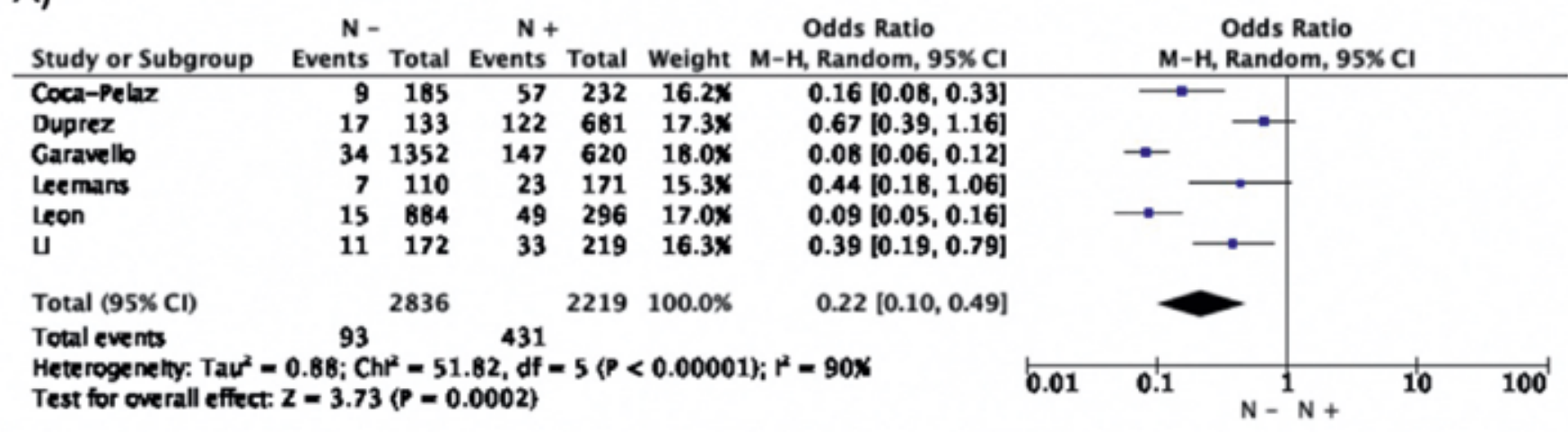

B)

$\begin{array}{cccc}\text { ECS - } & \text { ECS + } & \text { Odds Ratio } \\ \text { Study or Subgroup } & \text { Events } & \text { Total Events } & \text { Total Weight } \\ \text { M-H, Random, } 95 \% \mathrm{Cl}\end{array}$

\begin{tabular}{|c|c|c|c|c|c|c|}
\hline $\begin{array}{l}\text { Coca-Pelaz } \\
\text { Duprez } \\
\text { Garavello } \\
\text { Leon } \\
\text { U }\end{array}$ & $\begin{array}{l}18 \\
10 \\
52 \\
17 \\
14\end{array}$ & $\begin{array}{r}133 \\
84 \\
361 \\
125 \\
109\end{array}$ & $\begin{array}{l}33 \\
49 \\
85 \\
19 \\
19\end{array}$ & $\begin{array}{r}145 \\
153 \\
232 \\
66 \\
192\end{array}$ & $\begin{array}{l}20.3 \% \\
18.3 \% \\
24.5 \% \\
18.4 \% \\
18.5 \%\end{array}$ & $\begin{array}{l}0.53[0.28,1.00] \\
0.29[0.14,0.60] \\
0.29[0.20,0.43] \\
0.39[0.19,0.81] \\
1.34[0.64,2.80]\end{array}$ \\
\hline Total $(95 \% \mathrm{CI})$ & & 812 & & 788 & $100.0 \%$ & $0.46[0.27,0.78]$ \\
\hline Total events & 111 & & 205 & & & \\
\hline
\end{tabular}

Heterogenelty: Taur $^{2}=0.26 ; \mathrm{Ch}^{2}=14.43$, df $=4(\mathrm{P}=0.006) ; \mathrm{r}^{2}=72 \%$

Test for overall effect: $Z=2.86(P=0.004)$

Odds Ratio

$\mathrm{M}-\mathrm{H}$, Random, $95 \% \mathrm{Cl}$

C)

$<6 \mathrm{~cm} \quad>6 \mathrm{~cm} \quad$ Odds Ratio

\begin{tabular}{lrrrrrr} 
Study or Subgroup & Events & Total & Events & Total & Weight & M-H, Random, 95\% Cl \\
\hline Coca-Pelaz & 53 & 404 & 13 & 39 & $20.6 \%$ & $0.30[0.15,0.62]$
\end{tabular}

$\begin{array}{llllllll}\text { Duprez } & 121 & 969 & 13 & 45 & 24.0 \% & 0.35 & {[0.18,0.69]}\end{array}$

$\left.\begin{array}{lllllll}\text { Garavello } & 190 & 1894 & 23 & 78 & 41.8 \% & 0.27\end{array}\right]$

$\left.\begin{array}{lllllll}\text { Leon } & 57 & 1151 & 7 & 29 & 13.6 \% & 0.16\end{array}\right]$

$\begin{array}{lllll}\text { Total }(95 \% \mathrm{Cl}) & 4418 & 191 & 100.0 \% & 0.27\end{array}[0.20,0.38]$

Total events $421 \quad 56$

Heterogenelty: $\mathrm{Tau}^{2}=0.00 ; \mathrm{Ch}^{2}=1.90$, df $=3(\mathrm{P}=0.59) ; \mathrm{r}^{2}=0 \mathrm{X}$

Test for overall effect: $Z=7.72(P<0.00001)$

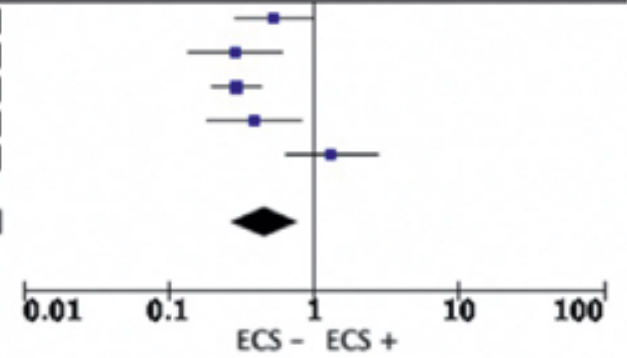

D)|

$\begin{array}{cccc} & <3 \text { Nodes }>3 \text { Nodes } & \text { Odds Ratio } \\ \text { Study or Subgroup Events Total Events Total Weight M-H, Random, } 95 \% \mathrm{Cl}\end{array}$ $\begin{array}{lllllll}\text { Coca-Pelaz } & 14 & 107 & 14 & 75 & \mathbf{3 0 . 5 \%} & 0.66[0.29,1.47]\end{array}$

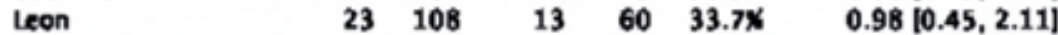

u

$\begin{array}{lllll}17 & 123 & 16 & 88 & 35.8 \%\end{array}$

$0.72[0.34,1.52]$

Total $(95 \% \mathrm{Cl})$

338

$223100.0 \%$

$0.78[0.50,1.21]$

Total events $54 \quad 43$

Heterogenelty: Taur $^{2}=0.00 ; \mathrm{Ch}^{2}=0.55$, df $=2(\mathrm{P}=0.76) ; \mathrm{r}^{2}=0 \mathrm{X}$

Test for overall effect $Z=1.11(P=0.27)$

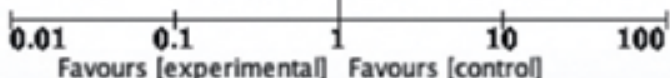

Figure 3. Forest plots showing the relationships between: (A) N-category; (B) Extranodal extension; (C) Lymph node size; (D) Number of lymph nodes and appearance of DM. The experimental cohort was represented by patients with negative lymph nodes, absence of ENE, lymph node $<6 \mathrm{~cm}$ in size, and less than 3 positive lymph nodes, aiming to demonstrate the protective effect of these factors on the development of DM. 


\section{f) Lymph node size}

After stratifying N0 and N+ patients, comparing DM appearance according to lymph node size $(\mathrm{N} 0$ or $\mathrm{N}+<6 \mathrm{~cm}$ versus $>6 \mathrm{~cm})$, the incidence of DM was $9.5 \%\left(\mathrm{CI}_{95 \%}\right.$ $8.6 \%-10.3 \%)$ versus $29.3 \% \quad\left(\mathrm{CI}_{95 \%} 22.85 \%-35.7 \%\right)$ $(\mathrm{p}=0.0001)$. These differences were significant with lymph node size $>6 \mathrm{~cm}$ as a risk factor for DM (OR $0.32 ; \mathrm{CI}_{95 \%} 0.23-0.44 ; \mathrm{p}=0.00001$ ) (Fig. 3C).

g) Number of lymph nodes

When stratifying $\mathrm{N}+$ patients according to the number of lymph nodes $(<3$ or $\geq 3)$, the incidence of DM was $15.9 \%\left(\mathrm{CI}_{95 \%} 12 \%-19.8 \%\right)$ versus $19.3 \%\left(\mathrm{CI}_{95 \%} 14.1 \%\right.$ $24.4 \%)(\mathrm{p}=0.361)$. These differences were not significant (OR 0.82; $\mathrm{CI}_{95 \%}$ 0.53-1.27; $\mathrm{p}=0.39$ ) (Fig. 3D).

\section{h) Histological differentiation}

When stratifying patients according to histological differentiation (well versus moderate versus poorly differentiated), the incidence of DM was $4.2 \%\left(\mathrm{CI}_{95 \%} 2.9 \%\right.$ $5.5 \%)$ versus $9.2 \%\left(\mathrm{CI}_{95 \%} 8.1 \%-10.2 \%\right)$ versus $16.9 \%$ $\left(\mathrm{CI}_{95 \%} 14.4 \%-19.4 \%\right)(\mathrm{p}=0.0001)$. These differences were significant, with DM being more common in patients with poorly differentiated tumours (Tab. IV).

\section{Discussion}

To the best of our knowledge, this study is the first systematic review and meta-analysis to investigate the clinical and histopathological factors related to the appearance of DM in $\mathrm{HN}$ SCC patients. In the diagnostic workup of primary HNSCC, DM screening may have two main but distinct objectives: detection of occult DM in patients without curative options, and counseling on prognosis and how to optimise quality of life in patients who are already symptomatic. Moreover, the presence of DM at baseline evaluation influences survival, treatment selection and management of locoregionally recurrent disease. However, it is important to understand that, in selected cases, depending on histology and natural history of the disease, appropriate treatment schedules for palliative (when not potentially curative) trials may be available.

Studies which fulfilled criteria to be included in our metaanalysis are in line with the comprehensive results of our study. In particular, Leemans et al. ${ }^{28}$ reported on the development of DM in 281 patients who underwent neck dissection and had locoregional control during follow up. Factors such as histologically proven lymph node metastasis, more than 3 positive lymph nodes, and the presence of ENE were associated with the highest risk for DM. Coca-Pelaz et al. ${ }^{7}$ analysed risk factors for development of DM in 443 patients with surgically treated primary HNSCC. Patients with poorly differentiated tumours, 3 or more positive lymph nodes and bilateral nodal metastases were associ- ated with the highest risk for development of DM. Duprez et al. ${ }^{22}$ reported that advanced $\mathrm{N}$ category, advanced stages, presence of ENE, tumour location in the hypopharynx and oropharynx with HPV-negative status, and locoregional persistent/recurrent disease were risk factors for DM. León et al. ${ }^{4}$ analysed the development of DM in 1,244 patients with oral cavity, pharyngeal and laryngeal SCC with apparently complete locoregional control, and found that $86 \%$ of DM appeared within the first 2 years after diagnosis of the primary tumour, and tumour location in the hypopharynx and supraglottis were independently associated with increased risk of DM. Li et al. ${ }^{6}$ in a study including 391 patients reported that the number of neck levels involved, level of tumour invasion and site of primary tumour were decisive risk factors in determining the development of DM. Finally, Garavello et al. ${ }^{5}$ in a study on 1,972 patients reported that the risk of DM was influenced by age, site of primary tumour, local and/or regional extension, grading and achievement of locoregional control.

\section{Rate of DM in HNSCC patients}

The rate of DM varies substantially in the literature depending on the type of study. As mentioned above, in population-based studies, Kuperman et al. ${ }^{11}$ and Liu et al. ${ }^{18}$ reported that the rates of DM in patients with HNSCC were $2.8 \%$ and $3.1 \%$, respectively. On the other hand, in clinical studies, DM rates vary from 4.2 to $23.8 \%{ }^{4,12-14,21,32-38}$, while in autopsy studies the frequency is usually higher, ranging between $37 \%^{7}$ and $57 \%{ }^{15-17,39-50}$. Such figures might be also influenced by the site of tumours considered: in fact, DM are significantly higher in patients affected by nasopharyngeal carcinomas, with incidences varying from $36 \%$ to $51 \%$ in autopsy studies ${ }^{51}$. These higher rates have been also clinically confirmed in two studies published by Palazzi et al. analysing the outcomes of patients with regionally and non-regionally advanced nasopharyngeal carcinomas, where the authors reported DM in $21 \%$ and $18 \%$ of patients, respectively ${ }^{52,53}$.

As previously highlighted by León et al. ${ }^{4}$, a higher frequency of DM in autopsies could be explained by the greater sensitivity in finding DM in this type of study. Risk of bias from autopsy data is mainly related to the fact that most patients with unfavourable oncologic evolution die in hospitals, and these patients have a greater tendency of harbouring DM. Moreover, autopsy studies are more prone to detect small and incidental DM whose real occurrence can be higher than that reported in clinical and population-based studies. However, the DM rate found in our meta-analysis $(9.6 \%)$ is similar to what has been reported in previous clinical papers, and higher than those in population-based studies. 


\section{Clinical and histopathological factors related to DM}

According to our results and the previous studies mentioned above, poorly differentiated tumours, hypopharyngeal location, T3-T4 categories, presence of metastatic lymph nodes larger than $6 \mathrm{~cm}$ or with evidence of ENE and locoregional persistence or failure after treatment significantly increase the risk of developing DM. However, we were not able to find a significant impact of the presence of more than 3 positive lymph nodes and age on the risk of DM. Finally, we judged the overall quality of the evidence to be moderate, since the OCEBM graded all studies as Level B (2c) and the ensuing findings related to some factors remain uncertain, which should be addressed more carefully in future research.

\section{Radiological implications in HNSCC and DM}

Histopathological risk factors were based on postoperative specimen examination and were thus not available for pretreatment decision making. This can be a drawback in selection of patients for DM pretreatment screening, in which clinical risk factors would be much more valuable. However, modern radiological advances allow even more detailed investigation during preoperative diagnostic workup. In fact, as recently highlighted by de Bree et al. ${ }^{2}$, some validated clinical-radiological high-risk factors are bilateral as well as more than 3 lymph node metastases, lymph node metastases of $6 \mathrm{~cm}$ or larger, low jugular lymph node metastases, regional recurrence and second primary tumours ${ }^{25}$. Other reported factors of increased risk for DM development like T4 category and/or N2-N3 status, oropharyngeal, hypopharyngeal, and supraglottic sites, levels IV and VB lymph nodes involvement and radiologic signs suspicious for ENE are all within the detection capability of modern imaging ${ }^{29-31}$.

\section{Organs affected by DM}

The results from our meta-analysis are consistent with those described in the literature, being the lung, bone, liver and skin $(61.8 \%, 15.3 \%, 7.7 \%$, and $7.1 \%$, respectively) the most common sites for DM in our study population. This represents the rationale for routine PET/CT or neck and chest CT in HNSCC patients with the abovementioned risk factors. The main argument in favour of chest CT instead of PET/CT, apart from a more favourable cost-effectiveness ratio and widespread geographic availability, is that the former would likely capture DM in the chest, cervical or thoracic spine, and in part of the liver. Combining these advantages, it is probable that chest CT should be viewed in terms of its overall superiority over PET/CT for detection of DM in the HNSCC population ${ }^{18}$.

In a study by Jäckel et al. ${ }^{13}$ on 1,087 patients with newly diagnosed SCC of the upper aerodigestive tract, the lung
(68.5\%), liver (23.8\%) and bones (20\%) were the most common sites of DM. Concerning the incidence of intracranial metastases, the rates reported are around $0.4 \%$. Moreover, brain metastases are detected much more frequently (2-8\% of patients) if another DM is already present ${ }^{54}$.

The incidence of skin metastasis in HNSCC is reported to be between $1 \%$ and $2 \%$ and accounts for $10-15 \%$ of the overall DM burden. Pitman and Johnson ${ }^{55}$ reported that rate of skin DM was $0.76 \%$, an incidence consistent with the results of other studies ${ }^{14}$, accounting for $10 \%$ of the DM load. It could be argued that regional skin metastases are, in fact, caused by changed lymphatic drainage patterns following locoregional treatment. However, skin of the head, neck, and chest are also common locations for metastasis from primary tumours arising outside the head and neck ${ }^{56}$. Finally, there is some debate about DM in the mediastinum, because these could also be lower extensions of regional metastases, as in the case of hypopharyngeal cancer, since the boundaries between VI and VII levels are notoriously ill-defined and wandering. In addition, occult second primary lung cancers may be responsible for this, especially if suboptimal diagnostic workup is performed.

Regarding DM treatment, the evidence for metastasectomy in HNSCC is still controversial, and large prospective studies are needed. A systematic review reported Level $2 \mathrm{a}$ evidence of the effectiveness of pulmonary metastasectomy for metachronous DM in HNSCC ${ }^{57}$. However, evidence for liver metastasectomy in HNSCC is scarce ${ }^{58}$. In a retrospective analysis recently published by Schultz et al. ${ }^{59}$, the authors demonstrated a significant survival benefit for $\mathrm{HN}$ SCC patients who received specific treatments (surgery or RT) for DM regardless of their origin. They also described significantly worse outcomes in patients with metastases to multiple organs, underlying the importance of treating mainly oligometastatic and/or single metastatic clinical scenarios. Other approaches to disease eradication, such as stereotactic body RT, have also been used to treat one or a limited number of pulmonary metastases ${ }^{60}$. However, long-term follow-up data are even more limited than that available for surgical resection.

\section{Oropharyngeal HPV-related carcinoma}

Among the studies included in this meta-analysis, only Duprez et al. ${ }^{22}$ reported the incidence of HPV in HNSCC. Therefore, at the moment, we were not able to extract any new conclusions about the relationships between HPV status and the risk of DM. From our systematic review, the evidence from the current literature is too heterogeneous to draw any meaningful assumptions about this aspect.

Regarding the identification of risk factors associated with a higher incidence of DM in HPV-positive oropharyngeal 
SCC, Weller et al. ${ }^{61}$ found that, among these patients, those with T4 tumours and/or active smokers had substantial rates of DM. They further reported an increased rate of DM in patients treated with cetuximab compared with those managed by cisplatin.

More recently, in a systematic review and meta-analysis by Tiedemann et al. ${ }^{62}$, time to DM following primary treatment of patients with HPV-positive SCC appeared to be longer, with metastases more likely to disseminate to more than two organs compared to patients with HPV-negative oropharyngeal tumours.

\section{Perspectives}

One of the most intriguing issue related to the DM topic in HNSCC comes from the question of a real need for routine screening of asymptomatic patients during follow-up. The evidence to date has a limited clinical value since DM from HNSCC usually cannot be treated with curative intent, and asymptomatic DM may not require immediate palliative treatment ${ }^{4,9,34,35,63}$. Importantly, DM usually appear shortly after treatment, with typical curves during follow-up showing a rapid increase between months 0 and 8 after treatment, with a subsequent slow increase between months 8 and 24 , and a substantial plateau between months 24 and 84 , indicating the absence of late metastasis ${ }^{64,65}$. This is in contrast with other types of tumours in the head and neck area such as, for example, adenoid cystic carcinoma, which frequently metastasises late in the clinical history of patients ${ }^{22}$.

\section{Limitations}

Due to its overall design, this meta-analysis presents an absence of uniformity and high heterogeneity across studies due to the retrospective nature and lack of randomization in the papers included. From this drawback derives also a low level of evidence extracted from them. Attempts were made to reduce bias and increase the study's validity by using the OCEBM grading system and including only studies with Level B (2c) evidence. According to the ROBIN-I, overall bias evaluation was considered to be at low to moderate risk in most studies, where the main reason for lowering the quality was the risk of bias due to missing data (differential loss to follow up affected by prognostic factors), measurement of outcomes (differential or non-differential errors in measurement of outcome data) and selection of the results depending on findings (Tab. V). Although this significantly minimised the potential for bias, it cannot be excluded.

Another limitation comes from the estimation of the real number of DM. All studies without autopsy confirmation probably underestimated the actual rate of DM, especially if they date before the radiology expansion era of the last three decades. Moreover, as different authors have hypothesised ${ }^{22}$, in previous times a less aggressive locoregional treatment might have been used for patients who were more likely to die from locoregional recurrence before DM could even become clinically apparent. Finally, we need to highlight as another potential study limitation, the lack of adequate information about nasopharyngeal cancer in almost all the studies included.

\section{Conclusions}

According to the results of the present meta-analysis, hypopharyngeal site, advanced $\mathrm{T}$ and $\mathrm{N}$ categories, ENE, lymph node size $>6 \mathrm{~cm}$, locoregional failure and poorly differentiated histology significantly increase the risk of developing DM in patients with HNSCC. Imaging can play a relevant role in the diagnostic work-up of these patients as it evaluates clinical and radiological factors related to DM and, possibly, can help modulate palliative/curative management. Larger studies comparing the risk of DM in HPV-positive and negative oropharyngeal SCC are needed.

\section{References}

1 Takes RP, Rinaldo A, Silver CE, et al. Distant metastases from head and neck squamous cell carcinoma. Part I. Basic aspects. Oral Oncol 2012;48:775-9. https://doi.org/10.1016/j.oraloncology.2012.03.013

2 de Bree R, Senft A, Coca-Pelaz A, et al. Detection of distant metastases in head and neck cancer: Changing landscape. Adv Ther 2018;35:161-172. https://doi.org/10.1007/s12325-018-0662-8

3 Ferlito A, Shaha AR, Silver CE, et al. Incidence and sites of distant metastases from head and neck cancer. ORL J Otorhinolaryngol Relat Spec 2001;63:202-7. https://doi.org/10.1159/000055740

4 León X, Quer M, Orús C, et al. Distant metastases in head and neck cancer patients who achieved loco-regional control. Head Neck 2000;22:680-6. https://doi.org/10.1002/1097-0347

5 Garavello W, Ciardo A, Spreafico R, et al. Risk factors for distant metastases in head and neck squamous cell carcinoma. Arch Otolaryngol Head Neck Surg 2006;132:762-6. https://doi.org/10.1001/ archotol.132.7.762

6 Li X, Di B, Shang Y, et al. Clinicopathologic risk factors for distant metastases from head and neck squamous cell carcinomas. Eur J Surg Oncol 2009;35:1348-53. https://doi.org/10.1016/j.ejso.2009.06.010

7 Coca-Pelaz A, Rodrigo JP, Suárez C. Clinicopathologic analysis and predictive factors for distant metastases in patients with head and neck squamous cell carcinomas. Head Neck 2012;34:771-5. https:// doi.org/10.1002/hed.21804

8 Lim JY, Lim YC, Kim SH, et al. Predictive factors of isolated distant metastasis after primary definitive surgery without systemic treatment for head and neck squamous cell carcinoma. Oral Oncol 2010;46:5048. https://doi.org/10.1016/j.oraloncology.2010.02.005

9 Leibel SA, Scott CB, Mohiuddin M, et al. The effect of local-regional control on distant metastatic dissemination in carcinoma of the head and neck: results of an analysis from the RTOG head and neck database. Int J Radiat Oncol Biol Phys 1991;21:549-56. https://doi. org/10.1016/0360-3016(91)90669-u

10 Kowalski LP, Carvalho AL, Martins Priante AV, et al. Predictive fac- 
tors for distant metastasis from oral and oropharyngeal squamous cell carcinoma. Oral Oncol 2005;41:534-41. https://doi.org/10.1016/j.oraloncology.2005.01.012

11 Kuperman DI, Auethavekiat V, Adkins DR, et al. Squamous cell cancer of the head and neck with distant metastasis at presentation. Head Neck 2011;33:714-8. https://doi.org/10.1002/hed.21529

12 de Bree R, Deurloo EE, Snow GB, et al. Screening for distant metastases in patients with head and neck cancer. Laryngoscope 2000;110:397-401. https://doi.org/10.1097/00005537-20000300000012

13 Jäckel MC, Rausch H. Distant metastasis of squamous epithelial carcinomas of the upper aerodigestive tract. The effect of clinical tumor parameters and course of illness. HNO 1999;47:3844. https://doi. org/10.1007/s001060050356

14 Alvi A, Johnson JT. Development of distant metastasis after treatment of advanced-stage head and neck cancer. Head Neck 1997;19:500-5. https:// doi.org/10.1002/(sici)1097-0347(199709)19:6<500::aid-hed7>3.0.co;2-2

15 Nishijima W, Takooda S, Tokita N, et al. Analyses of distant metastases in squamous cell carcinoma of the head and neck and lesions above the clavicle at autopsy. Arch Otolaryngol Head Neck Surg 1993;119:658. https://doi.org/10.1001/archotol.1993.01880130067009

16 Zbären P, Lehmann W. Frequency and sites of distant metastases in head and neck squamous cell carcinoma. An analysis of 101 cases at autopsy. Arch Otolaryngol Head Neck Surg 1987;113:762-4. https:// doi.org/10.1001/archotol.1987.01860070076020

17 Kotwall C, Sako K, Razack MS, et al. Metastatic patterns in squamous cell cancer of the head and neck. Am J Surg 1987;154:439-42. https://doi.org/10.1016/0002-9610(89)90020-2

18 Liu JC, Bhayani M, Kuchta K, et al. Patterns of distant metastasis in head and neck cancer at presentation: implications for initial evaluation. Oral Oncol 2019;88:131-6. https://doi.org/10.1016/j.oraloncology.2018.11.023

19 Haigentz M Jr, Hartl DM, Silver CE, et al. Distant metastases from head and neck squamous cell carcinoma. Part III. Treatment. Oral Oncol 2012;48:787-93. https://doi.org/10.1016/j.oraloncology.2012.03.019

20 Vermorken JB, Mesia R, Rivera F, et al. Platinum-based chemotherapy plus cetuximab in head and neck cancer. N Engl $\mathrm{J}$ Med 2008;359:1116-27. https://doi.org/10.1056/NEJMoa0802656

21 Calhoun KH, Fulmer P, Weiss R, et al. Distant metastases from head and neck squamous cell carcinomas. Laryngoscope 1994;104:1199205. https://doi.org/10.1288/00005537-199410000-00003

22 Duprez F, Berwouts D, De Neve W, et al. Distant metastases in head and neck cancer. Head Neck 2017;39:1733-43. https://doi. org/10.1002/hed.24687

23 Peters TT, Senft A, Hoekstra OS, et al. Pretreatment screening on distant metastases and head and neck cancer patients: validation of risk factors and influence on survival. Oral Oncol 2015;51:267-71. https:// doi.org/10.1016/j.oraloncology.2014.12.006

24 Spector ME, Chinn SB, Rosko AJ, et al. Diagnostic modalities for distant metastasis in head and neck squamous cell carcinoma: are we changing life expectancy? Laryngoscope 2012;122:1507-11. https:// doi.org/10.1002/lary.23264

25 Uyl-de Groot CA, Senft A, de Bree R, et al. Chest CT and whole-body 18F-FDG PET are cost-effective in screening for distant metastases in head and neck cancer patients. J Nucl Med 2010;51:176-82. https:// doi.org/10.2967/jnumed.109.067371

26 Huang B, Law MW, Khong PL. Whole-body PET/CT scanning: estimation of radiation dose and cancer risk. Radiology 2009;251:16674. https://doi.org/10.1148/radiol.2511081300.

27 de Bree R, Ljumanovic R, Hazewinkel MJ, et al. Radiologic extranodal spread and matted nodes: important predictive factors for de- velopment of distant metastases in patients with high-risk head and neck cancer. Head Neck 2016;38(Suppl 1):E1452-8. https://doi. org/10.1002/hed.24257

28 Leemans CR, Tiwari R, Nauta JJ, et al. Regional lymph node involvement and its significance in the development of distant metastases in head and neck carcinoma. Cancer 1993;71:452-6. https://doi.org/10.1002/1097-0142(19930115)71:2<452::aidcncr2820710228>3.0.co;2-b

29 Ljumanovic R, Langendijk JA, Hoekstra OS, et al. Distant metastases in head and neck carcinoma: identification of prognostic groups with MR imaging. Eur J Radiol 2006;60:58-66. https://doi.org/10.1016/j. ejrad.2006.05.019

30 Haerle SK, Schmid DT, Ahmad N, et al. The value of (18)F-FDG PET/ CT for the detection of distant metastases in high-risk patients with head and neck squamous cell carcinoma. Oral Oncol 2011;47:653-9. https://doi.org/10.1016/j.oraloncology.2011.05.011

31 Loh KS, Brown DH, Baker JT, et al. A rational approach to pulmonary screening in newly diagnosed head and neck cancer. Head Neck 2005;27:990-4. https://doi.org/10.1002/hed.20261

32 Rubenfeld S, Kaplan G, Holder AA. Distant metastases from head and neck cancer. Am J Roentgenol Radium Ther Nucl Med 1962;87:441-8.

33 Berger DS, Fletcher GH. Distant metastases following local control of squamous cell carcinoma of the nasopharynx, tonsillar fossa, and base of the tongue. Radiology 1971;100:141-3. https://doi. org/10.1148/100.1.141

34 Probert JC, Thompson RW, Bagshaw MA. Patterns of spread of distant metastases in head and neck cancer. Cancer 1974;33:12733. https://doi.org/10.1002/1097-0142(197401)33:1<127::AIDCNCR2820330119>3.0.CO;2-L

35 Merino OR, Lindberg RD, Fletcher GH. An analysis of distant metastases from squamous cell carcinoma of the upper respiratory and digestive tracts. Cancer 1977;40:145-51. https://doi.org/10.1002/10970142(197707)40:1<145::aid-cncr2820400124>3.0.co;2-9

36 Black RJ, Gluckman JL, Shumrick DA. Screening for distant metastases in head and neck cancer patients. Aust NZ J Surg 1984;54:527-30. https://doi.org/10.1111/j.1445-2197.1984.tb05440.x

37 Vikram B, Strong EW, Shah JP, et al. Failure at distant sites following multimodality treatment for advanced head and neck cancer. Head Neck Surg 1984;6:730-3. https://doi.org/10.1002/hed.2890060305

38 Bhatia R, Bahadur S. Distant metastasis in malignancies of the head and neck. J Laryngol Otol 1987;101:925-8. https://doi.org/10.1017/ s0022215100102993

39 Troell RJ, Terris DJ. Detection of metastases from head and neck cancers. Laryngoscope 1995;105:247-50. https://doi. org/10.1288/00005537-199503000-00005

40 Crile GW. Carcinoma of the jaw, tongue, cheek, and lips. Surg Gynecol Obstet 1923;36:159-84.

41 Price LW. Metastasis in squamous carcinoma. Am J Cancer 1934;22:1-6

42 Burke EM. Metastases in squamous cell carcinoma. Am J Cancer 1937;30:493-503.

43 Braund RR, Martin HE. Distant metastases in cancer of the upper respiratory and alimentary tracts. Surg Gynecol Obstet 1941;73:63-71.

44 Peltier LF, Thomas LB, Crawford TH, et al. The incidence of distant metastasis among patients dying with head and neck cancers. Surgery 1951;30:827-33.

45 Gowen GF, Desuto-Nagy G. The incidence of sites of distant metastases in head and neck carcinoma. Surg Gynecol Obstet 1963;116:6037. https://doi.org/10.1097/00006534-196310000-00028

46 Abramson AL, Parisier SC, Zamansky MJ, et al. Distant metastases 
from carcinoma of the larynx. Laryngoscope 1971;81:1503-11. https://doi.org/10.1288/00005537-197109000-00015

47 O'Brien PH, Carlson R, Steubner EA Jr, et al. Distant metastases in epidermoid cell carcinoma of the head and neck. Cancer 1971;27:304-7. https://doi.org/10.1002/10970142(197102)27:2<304::aid-cncr2820270209>3.0.co;2-1

48 Bruger J, Blache R, Cachin Y. Extension métastatique des épitheliomas épidermoides des voies aéro-digestives supérieures: Bilan de l'autopsie de 220 malades. Bull Cancer 1972;59:435-48.

49 Dennington ML, Carter DR, Meyers AD. Distant metastases in head and neck epidermoid carcinoma. Laryngoscope 1980;90:196-201. https://doi.org/10.1288/00005537-198002000-00002

50 Ahmad A, Stefani S. Distant metastases of nasopharyngeal carcinoma: a study of 256 male patients. J Surg Oncol 1986;33:194-7. https:// doi.org/10.1002/jso.2930330310

51 Palazzi M, Guzzo M, Bossi P, et al. Regionally advanced nasopharyngeal carcinoma: long-term outcome after sequential chemotherapy and radiotherapy. Tumori 2004;9:60-5.

52 Palazzi M, Guzzo M, Tomatis S, et al. Improved outcome of nasopharyngeal carcinoma treated with conventional radiotherapy. Int $\mathbf{J}$ Radiat Oncol Biol Phys 2004;60:1451-58. https://doi.org/10.1016/j. ijrobp.2004.05.059

53 Langley RR, Fidler IJ. The seed and soil hypothesis revisited - the role of tumor-stroma interactions in metastasis to different organs. Int J Cancer 2011;128:2527-35. https://doi.org/10.1002/ijc.26031

54 de Bree R, Mehta DM, Snow GB, et al. Intracranial metastases in patients with squamous cell carcinoma of the head and neck. Otolaryngol Head Neck Surg 2001;124:217-21. https://doi.org/10.1067/ mhn.2001.112478

55 Pitman KT, Johnson JT. Skin metastases from head and neck squamous cell carcinoma: incidence and impact. Head Neck 1999;21:5605. https://doi.org/10.1002/(sici)1097-0347(199909)21:6<560::aidhed $10>3.0 . c 0 ; 2-\mathrm{q}$

56 Schwartz RA. Cutaneous metastatic disease. J Am Acad Dermatol 1995;33:161-82. https://doi.org/10.1016/0190-9622(95)90231-7
57 Young ER, Diakos E, Khalid-Raja M, et al. Resection of subsequent pulmonary metastases from treated head and neck squamous cell carcinoma: systematic review and meta-analysis. Clin Otolaryngol 2015;40:208-18. https://doi.org/10.1111/coa.12348

58 Florescu C, Thariat J. Local ablative treatments of oligometastases from head and neck carcinomas. Crit Rev Oncol Hematol 2014;91:4763. https://doi.org/10.1016/j.critrevonc.2014.01.004

59 Schulz D, Wirth M, Piontek G, et al. Improved overall survival in head and neck cancer patients after specific therapy of distant metastases. Eur Arch Otorhinolaryngol 2018;275:1239-47. https://doi. org/10.1007/s00405-018-4920-9

60 Norihisa Y, Nagata Y, Takayama K, et al. Stereotactic body radiotherapy for oligometastatic lung tumors. Int J Radiat Oncol Biol Phys 2008;72:398-403. https://doi.org/10.1016/j.ijrobp.2008.01.002

61 Weller MA, Ward MC, Berriochoa C, et al. Predictors of distant metastasis in human papillomavirus - associated oropharyngeal cancer. Head Neck 2017;39:940-6. https://doi.org/10.1002/hed.24711

62 Tiedemann D, Jakobsen KK, von Buchwald C, et al. Systematic review on location and timing of distant progression in human papillomavirus-positive and human papillomavirus-negative oropharyngeal squamous cell carcinomas. Head Neck 2019;41:793-8. https://doi. org/10.1002/hed.25458

63 Arons MS, Smith RR. Distant metastases and local recurrence in head and neck cancer. Ann Surg 1961;154:235-40. https://doi. org/10.1097/00000658-196108000-00007

64 Dietl B, Marienhagen J, Schaefer C, et al. Frequency and distribution pattern of distant metastases in patients with ENT tumors and their consequences for pretherapeutic staging. Strahlenther Onkol 2007;183:138-43. https://doi.org/10.1007/s00066-007-1611-7

65 Calvin DP, Hammond ME, Pajak TF, et al. Microvessel density > or $=60$ does not predict for outcome after radiation treatment for locally advanced head and neck squamous cell carcinoma: Results of a correlative study from the Radiation Therapy Oncology Group (RTOG) 90-03 Trial. Am J Clin Oncol 2007;30:406-19. https://doi. org/10.1097/COC.0b013e3180342fd4 